\title{
Italique
}

Poésie italienne de la Renaissance

$X \mid 2007$

Varia

\section{Les livres italiens de Philippe Desportes}

\section{François Rouget}

\section{OpenEdition}

\section{Journals}

Édition électronique

URL : http://journals.openedition.org/italique/78

DOI : $10.4000 /$ italique.78

ISSN : 1663-4438

\section{Éditeur}

Librairie Droz

\section{Édition imprimée}

Date de publication : 1 septembre 2007

Pagination : 85-104

ISBN : 978-2-600-01156-3

ISSN : 1423-3983

Référence électronique

François Rouget, «Les livres italiens de Philippe Desportes », Italique [En ligne], X | 2007, mis en ligne le 01 septembre 2010, consulté le 19 avril 2019. URL : http://journals.openedition.org/italique/78 ;

DOI : 10.4000/italique.78 


$$
\text { FRANÇOIS ROUGET }
$$

LES LIVRES I T A L I EN S

DE PHILIP PE DES PORTES 



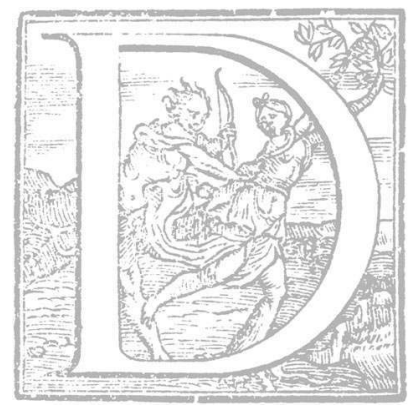

Epuis la publication des travaux de $H$. Vaganay, J. Vianey, et surtout ceux plus récents et décisifs de J. Balsamo, ${ }^{\mathrm{I}}$ on connaît mieux la nature et l'étendue des échanges culturels qui s'établirent au XVI ${ }^{e}$ siècle entre la France et l'Italie. Au cours de ces échanges, et étant donné l'attrait qu'exerça l'Italie sur la France, le livre italien joua une place importante et influença considérablement la littérature française. Dès les campagnes militaires de François $I^{\text {er }}$ en Italie, les Français acquirent de nombreux livres publiés en Italie, et les échanges ne cessèrent de se multiplier. ${ }^{2}$ A. Parent rappelle que pour répondre aux besoins croissants du public parisien en livres d'bumanités, les marchands et libraires français importent directement des livres de Venise et de Florence. De plus, les imprimeurs italiens - comme les héritiers d'Alde - «viennent eux-mêmes vendre leurs livres à Paris et y installer des dépôts». ${ }^{3}$

C'est dire que par les contacts directs ou indirects entre les divers partenaires du marché du livre, les lecteurs français peuvent aisément assouvir leur curiosité et leur goût pour la bibliophilie. L'attrait pour le livre italien ne se démentira pas tout au long du XVT ${ }^{e}$ siècle. On le constate à la lecture des inventaires des grandes bibliothèques du royaume, en particulier dans la seconde moitié du siècle. Dans une récente étude publiée dans cette revue, Isabelle de Conihout parvenait à chiffrer la proportion de livres italiens dans quelques bibliothèques d'amateurs: ${ }^{4}$ I6\% pour celle des Laubespine-Villeroy, $25 \%$ pour celle de Thomas Mabieu, 20\% pour celle de Florimond Robertet de Fresne, contre 6\% dans celle de Jacques-Auguste de Thou, autant dans celles de Claude Dupuy et de R. Belleau.' La bibliothèque d'écrivains de premier plan, comme Montaigne et Ph. Desportes, présente entre I6 et I8\% de livres italiens. C'est aussi la proportion que l'on retrouve dans la belle collection réunie par et pour Henri III, et sa scur Marguerite de Valois, à la même époque. ${ }^{6}$ La place des livres italiens dans les collections privées va dono croissant vers I570, indice de l'influence exercée par les Italiens à la cour de Henri III, et auprès de poètes français qui redécouvrent et illustrent le néopétrarquisme.

Parmi ceux-ci, Philippe Desportes constitue un cas exemplaire. Favori du roi qu'il suivit en Pologne, imitateur des pétrarquistes italiens, fin lecteur de littérature italienne, il parvint à réunir une bibliothèque riche qu'on peut évaluer à I0oo titres. Dans la recension effectuée par I. de Conihout, on identifie une quarantaine de livres en italien, mais c'est sans compter les livres en latin sortis des presses italiennes ni ceux en italien parus dans les autres pays d'Europe. ${ }^{7}$ 
Dans les pages qui suivent, nous voudrions rappeler l'histoire de la bibliothèque de Desportes, qui suscita l'admiration et la convoitise de ses contemporains, puis étudier la place qu'y occupent tous les livres italiens - qu'ils aient été ou non publiés en Italie - et signaler à l'attention des chercheurs de nowveaux titres italiens, non recensés jusqu'ici, et qui viennent grossir la bibliothèque de Desportes.

\section{Les livres en italien}

Nous savons par son biographe, J. Lavand, que Philippe Desportes possédait une fort belle bibliothèque dont il était fier, et dont l'histoire a été retracée par Isabelle de Conihout. ${ }^{8}$ La partie italienne était importante, ce qui n'étonne point quand on sait avec quelle ferveur Desportes imita Pétrarque, l'A rioste et les poètes néo-pétrarquiens du XV $T^{e}$ siècle. ${ }^{9}$ Le goût de Desportes pour l'italien est attesté très tôt par sa participation au volume d'Imitation de quelques chans de L'Arioste par divers poetes François (Paris, $L$. Breyer, I572) et par la composition de ses Premieres CEuvres, dont l'édition princeps est de I573. C'est aussi le moment où Desportes accompagne Henri d'Anjou et de Pologne dans son voyage polonais, et lors du retour, durant l'été de I574, Desportes le suit encore en qualité de secrétaire. Lors du séjour à Venise, Desportes en profita pour acquérir des livres que lui disputa le duc d'Anjou, nouveau roi de France. ${ }^{10}$ À l'image de son roi, qui se mit à l'étude de l'italien sous la conduite de J. Corbinelli et qui ne cessa d'accroitre sa bibliothèque, Desportes montra toujours une curiosité insatiable pour la littérature transalpine, curiosité qui se révèle dans le nombre de ses livres italiens. Selon l'inventaire dressé par I. de Conihout, ceux-ci figurent au nombre de 45 et constituent plus de $15 \%$ de sa bibliothèque. Il s'agit naturellement d'un total provisoire car d'autres titres italiens portant l'ex libris de Desportes peuvent faire l'objet de redécouvertes, comme on le verra plus loin. Par ailleurs, le chiffre avancé ne tient compte que des livres de langue italienne et laisse de côté tous les autres, ceux qui ont été écrits en latin (une quarantaine), ou traduits en langue moderne, à l'étranger (une quinzaine d'ouvrages). Tous ces livres font pourtant partie d'un ensemble ou «corpus» italien de Desportes qui a pu l'influencer à des degrés divers, que ce soit avant le départ pour la Pologne (I573) ou après son retour en France (I575), ${ }^{\mathrm{II}}$ et cela jusqu'à la fin de l'existence du poète chartrain. ${ }^{12}$

Ces livres italiens de langue italienne, entrés dans la collection de Desportes, recouvrent divers centres d'intérêt. La poésie y figure en bonne place: outre les grands classiques (Pétrarque; Dante, Convivio, I53I, $n^{\circ} 55$ ), Desportes a porté sa curiosité sur leurs commentateurs (J. Mazzoni, Della difesa della Comedia di Dante, I587, $n^{\circ}$ I89; G. Malatesta, Della nuova poesia 
overo delle difese del Furioso, I589, $n^{\circ}$ I96) et sur les poètes modernes, renommés (Serafino, Opere, I544, $n^{\circ} 74 ; \mathrm{F}$. Berni, Opere, I545, $n^{\circ} 77$ ) ou moins connus (C. Frangipani, Helice, I566, $n^{\circ}$ I3I; D. Rondinelli, Il Pastor vedovo, I599, $n^{\circ}$ 222). Signalons, enfin, que Desportes possédait un exemplaire du Commento [...] sopra piu sue Sonetti e canzoni (Florence, I500) de G. Benvieni, inconnu des bibliographes. ${ }^{\text {I3 }}$

Outre la poésie, les autres genres littéraires sont bien représentés, en particulier le genre épistolaire pour lequel Desportes semble avoir eu une véritable prédilection: A. S. Minturno, Lettere (Venise, I549, $n^{\circ}$ 87), L. Contile, Delle lettere (Pavie, I564, $n^{\circ}$ I27), B. Guarini, Lettere (Venise, I594, $n^{\circ} 211$ ) et G. B. Leoni, Delle lettere familiari (Venise, I600, $n^{\circ}$ 227). Exercice rhétorique illustrant l'éloquence de l'écrivain, la lettre connaît une vogue certaine vers Is6s et qui ne se démentira jusqu'au début du XVI siècle. Desportes, qui fréquente autant les «salons» de son époque que l'Académie du Palais, montre là l'étendue de sa curiosité. Possesseur de La Rhetorica de B. Cavalcanti (Venise, I578, $n^{\circ}$ I59), il peut aussi imiter l'éloquence que lui offrent les liures de discours: Discorsi de P. de Grimaldi Robio (Venise, I544, $n^{\circ} 76$ ) et Discorsi poetici de F. Summo (Padoue I600, $\left.n^{\circ} 226\right)$. Un autre témoignage de la curiosité de Desportes pour les questions linguistiques est étayé par la récente redécouverte de son exemplaire de L'Hercolano de B. Varchi (Florence, I570), où se voit débattue la question de l'usage littéraire de la langue toscane (fig. I). ${ }^{\mathrm{I} 6}$

Si le discours critique et le commentaire bistorique intéressent Desportes, c'est l'histoire en général qui retient son attention. Il peut ainsi goûter les Gioie historiche d'O. Toscanella (Venise, I567, n' I33) et les descriptions bistoriques de villes italiennes comme le Compendio dell'istoria [...] di Napoli par P. Collenuccio (Venise, I59I, $n^{\circ}$ 200) et l'Historia [...] di Milano par A. M. Spelta (Pavie, I603, $n^{\circ}$ 236). Signalons aussi La Historia della città di Parma de B. Angeli (Parma, I59I), dont Desportes possédait un exemplaire, non répertorié jusqu'ici (fig. 2-3). ${ }^{17}$ À côté de l'bistoire des villes et des principautés, Desportes attache un intérêt ponctuel à l'bistoire politique (S. Schiappalaria, Osservationi politiche [...] Vita di Cesare, Vérone, I600, $n^{\circ} 228$ ) qui rejoint son goût pour l'bistoire et la stratégie militaire (L. Gorgieri, Trattato della guerra, Pesaro, I548-I555, no 98; F. Patrizzi, La militia romana di Polibio, Ferrare, $\left.1583, n^{\circ} I 74\right)$.

La curiosité historique de Desportes le conduit aussi à collectionner d'autres ouvrages de sciences, à commencer par la cosmographie et la géographie (G. L. Anania, L'Universale fabrica del mundo, Venise, I576, $n^{\circ}$ I52; F. L. Vulcano, Vera et nuova descrittione di tutta Terra Santa, Naples, I563, $n^{\circ}$ I24), mais encore la géométrie. En ce sens, la vente aux enchères cher. Sotheby's, en avril 2002, de l'exemplaire de Desportes de La Prospettiva di 
Euclide [...] (Florence, Giunti, Is73; fig. 4) constitue une double révélation. L'intérêt du poète pour la science des nombres reste cependant fort limité, tout comme son goût de l'économie que la possession du Trattato del debito de P. Torelli (Parme, I596, $n^{\circ}$ 217) ne suffit à prouver.

Enfin, dans cet ensemble de livres italiens, une place importante revient aux ouvrages de philosophie et de théologie, ce qui ne peut surprendre quand on sait la proportion des livres religieux dans la production éditoriale de l'époque, et quand on considère la composante sacrée de la production poétique tardive de Desportes (paraphrase des Psaumes). ${ }^{18}$ Sur les rayons de la bibliothèque du Chartrain, on trouve ainsi Pic de la Mirandole (Le Sette sposizioni [...] Heptaplomeron, Pescia, I555, $n^{\circ}$ 99), Denys l'Aréopagite (Delli divini nomi, Venise, I563, n' I23), pour la philosophie, et des titres de politique ecclésiale (I. Chizzuola, Risposta [...] Paolo Vergerio del concilio publicata da Papa Pio IV, Venise, I562, $n^{0}$ II 8 ) ou d'histoire religieuse ( $G$. Bosio, Dell'istoria della sacra religione [...], Rome, I594, $n^{\circ}$ 208). L'intérêt de Desportes pour les affaires religieuses, que l'on constate dans la composante italienne de sa bibliothèque, est avéré et renforcé par la présence de nombreux autres ouvrages portant sur l'bistoire, la doctrine et les débats de l'Église, composés en latin et en français. ${ }^{19}$

\section{Les livres en latin}

Jusqu'ici, notre enquête s'est portée sur les ouvrages italiens publiés en Italie. Il faut la poursuivre avec tous ces livres dont la langue est le latin et dont le nombre avoisine celui des titres en vernaculaire comptabilisés dans la bibliothèque de Desportes. L'importance du latin dans la production éditoriale italienne de l'époque ne peut surprendre; elle est identique en France. Le latin demeure encore à cette époque la langue des clercs et des savants. Étant donné la nature des sujets et des disciplines constatée dans la collection du poète chartrain, la place et la proportion des livres en latin confirment nos attentes. Comme pour les livres en langue italienne, les livres en latin imprimés en Italie recouvrent une diversité de contenus et ont été produits entre I493 (M. Bosso, Recuperationes desulanae, Bologne, $n^{\circ} 7$ ) et I604 (L. de Cruz, Liber psalmorum [...], Naples, $n^{\circ}$ 240), c'est-à-dire peu avant la disparition de Desportes en I606.

Pour un poète français du XVI ${ }^{e}$ siècle, l'écriture lyrique profane et sacrée trouve une de ses principales sources d'inspiration dans la littérature latine et néo-latine. Lecteur du Canzoniere de Pétrarque, du Décaméron de Boccace, Desportes goûte et connaît fort bien aussi les productions latines de ces grands écrivains. Observateur des innovations littéraires des poètes italiens $d u$ $X V T^{e}$ siècle, il imite et adapte, comme Ronsard et les membres de la «Bri- 
gade», les poésies latines de P. Bembo, A. Navagero, L. Alamanni, G. Cotta, M. Molza, l'Arioste et J. Sannazar. Là encore, ce qui frappe dans cette catégorie de livres latins est leur extrême diversité de matières. En poésie, F. Sabeo (Epigrammatum [...] libri quinque, Rome, I556, $n^{\circ}$ I02) figure aux côtés des poètes rassemblés par $G$. Taigeto dans son anthologie (Carmina praestantium poetarum, Brescia, I565, $n^{\circ}$ I28), mais aussi de B. Botta (Davidias, Pavie, I573, $n^{\circ}$ I46; fig. 5). C'est aussi dans l'édition italienne procurée par R. Titi qu'il lit l'œuvre latine de Némésien (Bucolica, Florence, I590, $n^{\circ}$ 197).

Langue de savoir et de communication du savoir, le latin sert de support linguistique à de nombreuses disciplines. Dans la bibliothèque latine de Desportes, les Belles-Lettres sont bien représentées. Outre la poésie, Desportes apprécie les ouvrages traitant d'éloquence. Le genre de la lettre, si abondamment illustré en italien, continue de fleurir en latin (M. Bosso, Epistolarum, Venise, I502, $n^{\circ} 21$ ). C'est par ce genre littéraire - autant que le dialogue que les écrivains définissent les principes de l'art oratoire. Le latin demeure la langue d'investigation et de pédagogie pour les questions de linguistique, de rhétorique et d'éloquence. Desportes détenait un exemplaire des Tabulae rhetoricae de R. C. Angeli (Venise, I57I), ${ }^{20}$ mais encore les Orationes d'A. Navagero (Venise, I530, $n^{\circ}$ 52), l'édition par C. Sigonio des Fragmenta Ciceronis [...] (Venise, I560, $n^{\circ}$ II5), et les Trattati overo discorsi [...] (Venise, I57I, $n^{\circ}{ }_{142}$ ) ainsi que la Rhetorica (Venise, I578, $\left.n^{o} I 59\right)$ de B. Cavalcanti. ${ }^{21}$

Curieux de tout, on l'a vu, Desportes a collectionné de nombreux autres ouvrages en latin sur des sujets historiques et philosophiques. En histoire, Desportes s'intéresse autant à l'Antiquité qu'à son temps. Nous savons qu'il possédait un exemplaire du De mysteriis Aegyptorium de Jamblique (Rome, I556; fig. 6), ${ }^{22}$ mais aussi de la Descriptio [...] rerum germanicarum (Rome, I546, $n^{\circ} 81$ ), le Commentarius de Obelisco (Rome, I586, $n^{\circ}$ 188) ${ }^{23}$ et les Annales de L. Cavitelli (Crémone, I588, $n^{\circ}$ 194). Histoire des peuples et des villes, l'bistoire investit aussi le champ de l'art militaire (P. Belli, De re militari et bello tractatus, Venise, I563, $n^{\circ}$ I2I), de l'architecture (éd. D. Barbaro du De Architectura de Vitruve, Venise, I567, $n^{\circ}$ 132), voire de la jurisprudence (C. Tolomei, De corruptis verbis juris civilis dialogus, Sienne, v. I5I7, $n^{\circ}$ 34; A. Massa, De exercitatione jurisperitorum libri tres, Rome, I550, $n^{\circ} 89$ ).

En philosophie, on retrouve Denys l'A réopagite dans la traduction que M. Ficin a réalisée $d u$ De Mystica theologia et du De Nominibus (Venise, I538, $n^{\circ}$ 64), les commentaires d'A. de Salo Scaino (In octo Arist. de Republ. Questiones, Rome, I577, $n^{\circ}$ 157) et le traité de P. Duodo (Peripateticarum de anima, Venise, $1587, n^{\circ}$ 192) sur la philosophie politique et 
métaphysique d'Aristote. Ces titres, auxquels il faut ajouter les manuscrits possédés par Desportes, ${ }^{24}$ corroborent l'intérêt que celui-ci portait à la philosophie.

On peut en dire autant de ses livres de doctrine chrétienne, qu'ils concernent les ouvrages de polémique, d'histoire, de traduction ou de commentaire. C'est cette catégorie d'ouvrages religieux qui est la plus nombreuse dans la collection de livres latins de Desportes; rien de très surprenant à cela, lorsque l'on garde à l'esprit que Desportes avait reçu les ordres mineurs, lui permettant de jouir de bénéfices ecclésiastiques, nombreux et richement dotés, et qu'il consacra la dernière partie de sa vie à paraphraser en français les Psaumes de la Bible. S'il dut utiliser nombre d'ouvrages de piété publiés en France (on songe en particulier au Psalterium sextuplex Hebraicum, Lyon, Gryphe, Is30, dans la traduction de S. Pagnino, $n^{\circ}$ sI), il put s'inspirer de la lecture du $\mathrm{De}$ confutatione hebraicae sectae de G. B. Graziadei (Rome, I500, no I 8), du Soliloquiae de C. Appiano (Crémone, I496, $n^{\circ}$ I2), des ouvrages des Pères et docteurs de l'Église, tels les Carmina de Grégoire de Nazianze (Venise, I504, $n^{\circ}$ 24), l'Ortus deliciarum de Laurent Justinien (Milan, ISIS, $n^{\circ}$ 30), les Opera de saint Thomas (Rome, I570, $n^{\circ}$ I40), et enfin des livres d'enseignement et de liturgie, comme les Institutiones christianae [...] de J. de Dambouder (Venise, I597, $n^{\circ} 218$ ), les Regulae omnes ordinationes [...] Pauli III (Rome, I547, $n^{\circ} 82$ ), ${ }^{25}$ le De consideratione et judicio praesentium temporum de L. Politi (Venise, I547, $n^{\circ} 83$ ) et le Psalmodia et catholicum Psalmorum [...] de B. Botta (Pavie, I590, $n^{\circ}$ I64). Au total, on constate que Desportes a été un découvreur et un lecteur attentif des ouvrages de piété publiés en latin dans l'Italie de la Renaissance.

\section{Livres italiens publiés hors d'Italie}

Il reste à porter un regard sur tous ces autres livres d'écrivains italiens qui furent publiés dans le reste de l'Europe. Dans ce domaine aussi, l'influence de la culture italienne se fit sentir de manière significative. Qu'ils publient des éditions originales ou des rééditions, en langue italienne, en latin ou en traduction, les éditeurs européens accueillent largement dans leurs fonds ces écrivains italiens, célèbres ou moins connus, que le public goûte alors avec ferveur.

On peut classer ces livres italiens publiés en Europe - ayant appartenu à Desportes - en fonction de leur lieu d'édition. On observe ainsi que la majorité d'entre eux (70\%) parurent en France. C'est d'abord de Lyon, plaque tournante du commerce et carrefour des échanges culturels entre l'Italie et la France, ò̀ s'était établie une importante communauté italienne, que proviennent les livres italiens de Desportes. Cette abondance est due en partie par l'impression et la diffusion de livres en langue italienne entreprises par les 
éditeurs lyonnais, comme Il Decamerone de Boccace, annotépar Bembo (G. Roville, I555; $n^{\circ}$ 96). ${ }^{26}$ Les livres d'auteurs italiens publiés à Lyon et retrouvés dans la collection de Desportes concernent surtout la théologie. Il s'agit du Tractatus de synodo episcopi (J. David, I529) ${ }^{27}$ de E. Botteo, du Psalterium sextuplex hebraicum (S. Gryphe, I530; $n^{\circ}$ II) de S. Pagnino, déjà mentionné, des Annotationes in Commentaria Caietani (M. Bonbomme, I542; $\left.n^{\circ} 69\right)$ de L. Politi, et du Libri de potestate Papae et concilii de G. G. Albani (J. de Tournes et G. Gazeau, I5s\&; $n^{\circ}$ 107).

Dans les ouvrages italiens publiés à Paris et collectionnés par Desportes, on constate une plus grande variété de sujets: la poésie néo-latine avec G. Pavini (Baculus pastoralis, F. Regnault, ISI4; $n^{\circ}$ 29) et l'anthologie procurée par G. M. Toscano (Carmina illustrium poetarum italorum, A. Gorbin, I576-I577; $n^{\circ}$ I54); l'bistoire littéraire avec l'importante édition du De Vulgari eloquentia de Dante commentée par Jacopo Corbinelli (J. Corbon, I577; $n^{\circ}{ }^{156)}$ - dont la parution cö̈ncide avec la promotion d'une éloquence royale soutenue par Henri III $-;^{28}$ l'art de la guerre avec le De re militari de R. Valturio (Chr. Wechel, Is35; $n^{\circ}$ 60); la philosophie avec le Spaccio de la bestia trionfante de G. Bruno (s. n., I584; $n^{\circ}$ I78); enfin, l'histoire ecclésiastique avec l'ouvrage de G. C. Romano, De Regimine principum ecclesiasticorum opusculum (Th. Brumen, $1587 ; n^{\circ}$ 193).

Produits dans la capitale du royaume dans laquelle résidaient le plus souvent la Cour et Desportes, ces livres italiens proposés aux chalands bénéficiaient d'une situation économique et culturelle privilégiée. Pour un poète italianisant comme Desportes, curieux des dernières parutions, cette production italienne d'auteurs italiens, à Lyon ou à Paris, en visite ou en résidence permanente à la Cour, comblait ses attentes et enrichissait ses goûts.

C'est sans doute encore à Paris que Desportes acquit les autres ouvrages italiens de sa collection, qu'ils aient été imprimés à Anvers, Londres, Hambourg, ou Bâle. Pour nombre des livres édités dans ces villes, l'un des lieux privilégiés de diffusion était la capitale française. Et, on le sait, certains titres sortis des presses anversoises étaient uniquement destinés au marché français. Les éditions anglaises figurant dans la bibliothèque de Desportes sont peu nombreuses: outre The Principall navigations (Londres, G. Bishop, Is 89) de R. Hakluyt-offert à Desportesparl'auteur lors de son séjourparisien?-, ${ }^{29}$ on note deux exemplaires $d u$ De legationibus libri tres de l'Italien A. Gentile (Londres, Th. Vautrollerius, I585; $n^{\circ}$ 179-180). D'Allemagne, plus précisément des presses hambourgeoises de J. Wolff, est sorti le traité de la Magia philosophica de F. Patrizzi (I593; $n^{\circ}$ 206). Deux autres ouvrages latins, le De bono senectutis de G. Paleotti (I598; $n^{\circ}$ 219), et les Poemata de L. Gambara $(I 569)^{30}$ sont sortis des presses plantiniennes à Anvers. Ajoutons, pour finir, deux titres en latin d'écrivains italiens édités à Bâle: il y a 
d'abord l'édition de Longin par F. Robortello (Liber de [...] orationis genera, J. Oporin, I554; $n^{\circ}$ 95). Signalons enfin un titre inconnu des recensions effectuées par les bibliophiles de Desportes: le volume des Epistolarum libri octo du célèbre bumaniste d'Arez२o, Leonard Bruni (H. Petri, I535; fig. 7), ${ }^{3 \mathrm{I}}$ dans la seule édition parue au XVI ${ }^{e}$ siècle de ce livre publié pour la première fois en 1472 (Venise, A. Moreto et G. Squarciafico), et qui constitue une source de première main pour connaître l'histoire politique, culturelle et littéraire de l'Italie du Quattrocento.

On le voit, Desportes semble avoir recherché puis accumulé dans son impressionnante bibliothèque toutes sortes d'ouvrages, des plus communs aux plus rares, lui permettant d'enrichir sa connaissance de l'Italie, de son histoire et de sa culture, et de se familiariser avec les plus notables personnages littéraires que l'Italie avait produits. La ferveur que montre Desportes pour la culture italienne fut reconnue par ses contemporains qui, non sans malice, se plurent à identifier les sources de ses poèmes assimilées à des plagiats. ${ }^{32}$ D'autres admirateurs de son cuvre, parmi lesquels figurent des Italiens (I. Andreini, $V$. Belando, Fl. De Birague, M. Equicola), ${ }^{33}$ surent lui rendre un vibrant hommage et choisirent même - tel M. Baccellini - de traduire dans la langue italienne celui qui avait tant fait pour acclimater au goût français la littérature italienne. $^{34}$

François Rouget 


\section{Les livres italiens de Philippe Desportes}

I. H. Vaganay, Le sonnet en France et en Italie au XVI siècle, Lyon, I903; J. Vianey, Le pétrarquisme en France au XVI ${ }^{e}$ siècle, Paris-Montpellier, I909; J. Balsamo, Les rencontres des Muses. Italianisme et anti-italianisme dans les Lettres françaises de la fin du XVT ${ }^{e}$ siècle, Genève, Slatkine, 1992.

2. Sur ce point, voir le rôle décisif joué par les rois français dans l'acquisition et l'importation en France de livres italiens qui servit de modèle aux autres lettrés; cf. U. Baurmeister, D'Amboise à Fontainebleau: les imprimés italiens dans les collections royales aux $X V^{e}$ et $X V T^{e}$ siècles, in Passer les monts. Français en Italie - l'Italie en France (I494-I525), Actes du X $\mathrm{X}^{\mathrm{e}}$ colloque de la Société française d'étude du Seizième Siècle, réunies par J. Balsamo, Paris-Florence, Champion-Cadmo, i 998, pp. 36 I-86.

3. A. Parent, Les métiers du livre à Paris au XVI siècle (I535-I560), Genève, Droz, I974, p. I 54.

4. I. de Conihout, $\grave{A}$ propos de la bibliothèque aux cotes brunes des Laubespine-Villeroy: les livres italiens chez. les secrétaires du roi dans la seconde moitié $d u X V T^{e}$ siècle, in «Italique», vol. VII (2004), pp. 137-59, voir en particulier les pp. I 52-55.

5. M. Connat-Jurgens, Mort et testament de Rémy Belleau, in «Humanisme et Renaissance», t. VI (1945), pp. 328-56.

6. Voir J. Boucher qui dresse un inventaire incomplet des ouvrages ayant appartenu au roi dans Société et mentalités autour de Henri III, Lille-Paris, ART - Champion, I98 I, t. III, pp. 86I-77. Voir F. Le Bars, Les reliures de Henri III: essai de typologie, in Henry III mécène dir. I. de Conihout, J.-F. Maillard et G. Poirier, Paris, Presses de l'Université de Paris-Sorbonne, 2006, pp. 228-47, et M.-N. Matuszeck, La bibliothèque de Marguerite de Valois, in Henry III mécène cit., pp. 274-92. M.-N. Matuszek dénombre une trentaine de livres italiens sur près de 300 titres.

7. I. de Conihout, Du nouveau sur la bibliothèque de Philippe Desportes et sur sa dispersion, in Philippe Desportes (1546-1606). Un poète presque parfait entre Renaissance et Classicisme, Actes du colloque international de Reims, ét. réunies par J. Balsamo, Paris, Klincksieck, 2000, pp. I 2 I -6o.

8. Voir respectivement J. Lavaud, Un poète de cour au temps des derniers Valois. Philippe Desportes (I546-1606), Paris, Droz, I936, p. 408 et suiv., et de Conihout, Du nouvean cit., pp. I 28-36.

9. Outre Lavaud, Un poète de cour cit., p. 4Io, voir Balsamo, Les rencontres des Muses cit., p. 232 et suiv., et F. Rouget, Philippe Desportes, médiateur du pétrarquisme français, in Les Poètes français de la Renaissance et Pétrarque, ét. réunies par J. Balsamo, Genève, Droz, 2004, pp. 33 I-5 I.

Io. Lavaud (Un poète de cour cit., pp. 224-25, note 4) rapporte à ce sujet une anecdote consignée par N. Bourbon (Borboniana, t. II, p. 304): «Le Roi étant à Venise, à son retour de Pologne, M. Des Portes y acheta pour cinq cens écus de livres. Le Roi les voulut avoir, et lui en fit rendre mille écus, et quelque temps après lui fit rendre les livres aussì.

I I. On note que la moitié des livres italiens de Desportes ont été publiés avant I 573, mais rien n'autorise à penser évidemment qu'ils sont entrés dans sa bibliothèque à cette date.

I 2. L'édition italienne la plus ancienne est celle des poésies profanes de Pétrarque (Milan, I 494; $\mathrm{n}^{\circ}$ Io de la liste de Conihout); l'une des dernières acquisitions est l'Historie di quattro principali citta del mondo de M. Zappullo (Vicence, I603; n 238 ).

I 3. Je remercie J. Balsamo de m'avoir indiqué l'existence de ce volume figurant dans la collection privée d'un Turinois. 
I4. Desportes possédait aussi un exemplaire de L'Amore innamorato (Venise, I $559, \mathrm{n}^{\circ}$ I I I) du même auteur.

I 5. Simultanément, Desportes semble avoir acquis Il Segretario du même Guarini, publié la même année ( $\mathrm{n}^{\circ}$ 2 1 2).

I6. Volume proposé par la Librairie Giraud-Badin, à Paris (2003; nº I Io).

I7. Ce volume a conservé sa reliure de l'époque aux armes et chiffres dorés d'Antoine de Selve. Nous remercions P. Sourget, de la Librairie Sourget, à Paris, de nous avoir procuré les deux clichés de cet ouvrage.

I 8. À ce sujet, on consultera l'introduction de B. Petey-Girard à sa belle édition des $C L$ Pseaumes de David mis en vers françois par Desportes (Paris, STFM, 2006, pp. 5-I I 8).

I9. Parmi les plus importants, citons le volume factice $n^{\circ} 44$ du répertoire d'I. de Conihout, comportant 2 I pièces latines publiées entre I 5 I 9 et I 526 , mais aussi un volume factice non signalé jusqu'ici et rassemblant 5 titres de Th. De Bèze dont le premier est le recueil de Sermons (Genève, Le Preulx, I 593) (Bibl. de la Sorbonne, TRP-22-I 2). Signalons enfin un recueil factice, absent du répertoire d'I. de Conihout, composé de livres exposant la vie et les doctrines des penseurs protestants (De persona et doctrina Martini Lutheri [...], par A. Catharinus, Mayence, I 548; etc.) et catalogué à l'Arsena de Toulouse ( $8^{\circ}$ Rés. Mn 6000).

20. Cet exemplaire, absent de la recension dresssée par I. de Conihout, figure à présent dans la Réserve de la Bibliothèque municipale de Chartres.

2I. Signalons que le volume factice, dans lequel ces deux titres ont été rassemblés (Bibliothèque Sainte-Geneviève, $4^{\circ}$ R. 587), contient aussi trois autres ouvrages de J. de Tenores, dont l'Introduttione [...] sopra i tre libri della rhetorica di Aristotile (Venise, I 578); ceux-ci, néanmoins, ne portent pas l'ex libris de Desportes.

22. Ce volume - non recensé jusqu'ici - est passé dans la collection des livres d'Antoine Godeau, un des admirateurs fervents de Desportes et, comme lui, paraphraste des Psaumes.

23. À noter que ce titre est rassemblé avec sept autres titres dans le recueil factice conservé à la Bibliothèque Mazarine (Maz. I 8355 ). Si un seul porte la signature de Desportes, on peut s'interroger cependant sur la provenance des autres qui présentent avec lui de nombreux points communs: lieu d'impression (Rome), date (six sur huit sont de I 586), imprimeur (Grassi pour 5 titres), sujet (l’obélisque de Rome, dans quatre titres).

24. Voir l'article d'I. de Conihout, $\grave{A}$ propos cit., liste des manuscrits, no 246-286, pp. I 5 5-60.

25. À noter que le recueil factice où se trouve ce titre en comporte deux autres sur le même sujet: Regula sanctissimi D. N. D. Pauli Papae Tertii [...], I 549, et Regula cancellariae sanctissimi Domini nostri Domni Pauli [...], I 548 (Bibliothèque Sainte-Geneviève, $8^{\circ}$ E. 3079, Inv. 1684). Mais seules les Regulae (I 547), en tête du volume, présentent la signature de Desportes et des traits de soulignement qui sont le signe d'une lecture attentive par le poète chartrain.

26. Sur ce point, voir N. Zemon Davis, Le monde de l'imprimerie humaniste: Lyon, in Histoire de l'édition française, t. I: Le livre conquérant du Moyen Age au milieu du XVII ${ }^{e}$ siècle, dir. H.-J. Martin et R. Chartier, Paris, Promodis, I982, pp. 25 5-77.

27. Recensé mais non localisé par I. de Conihout, ( $\left.n^{\circ} 48\right)$, cet ouvrage figure à présent à la Houghton Library de Harvard University (cote: IC 5. B6583. $573 \mathrm{~d}$ ). 


\section{Les livres italiens de Philippe Desportes}

28. Voir M. Fumaroli, Aulae Arcanae. Rhétorique et politique à la Cour de France sous Henri III et Henri IV, in «Journal des savants» (avril-juin I98I), pp. I37-89 (repris dans La diplomatie de l'esprit, Paris, Hermann, I994, pp. 59-I 24).

29. Absent de la liste d'I. de Conihout, l'ouvrage figure à présent dans la Réserve de la bibliothèque de Princeton University.

30. Inconnu d'I. de Conihout, cet exemplaire de Desportes a été vendu par la Librairie Scheler à Paris en novembre 2004 (Catalogue 29, $\mathrm{n}^{\circ} 76$ ). Relié à la suite figurait le Themis dea, seu de lege divina, de S. W. Pighius (Anvers, Plantin, I 568), bibliothécaire du cardinal de Granvelle, qui consacre ici deux études d'archéologie romaine dont la première concerne la statue de Thémis en marbre acquise par le cardinal Pius de Carpi à Rome. Seul le premier titre porte la signature de Desportes.

3I. Le livre a été vendu par la librairie Gilhofer \& Ranschburg, à Lucerne, en avril 2005 .

32. Voir Le [sic] Rencontre des Muses de France et d'Italie (Lyon, Roussin, I604), où se voient reproduits 43 sonnets de Desportes et leurs sources italiennes.

33. Voir en particulier les Rime de I. Andreini (Paris, Cl. De Monstrœil, I603) où l'auteur adresse un sonnet à Desportes qualifié de «Sacro Vate del Pindo»; et les Lettere facete de V. Belando (Paris, A. L'Angelier, I 588 ) qui saluent deux fois Desportes «appolinesca e prior del Monte Parnasso».

34. Voir Li Sette Salmi penitentiali Davidici. Aggiunti li salmi penitentiali Franzesi della tradottione del dottissimo Signor Filippo Portes abbate di Tirrone (Paris, Ph. Du Pré, I604). 


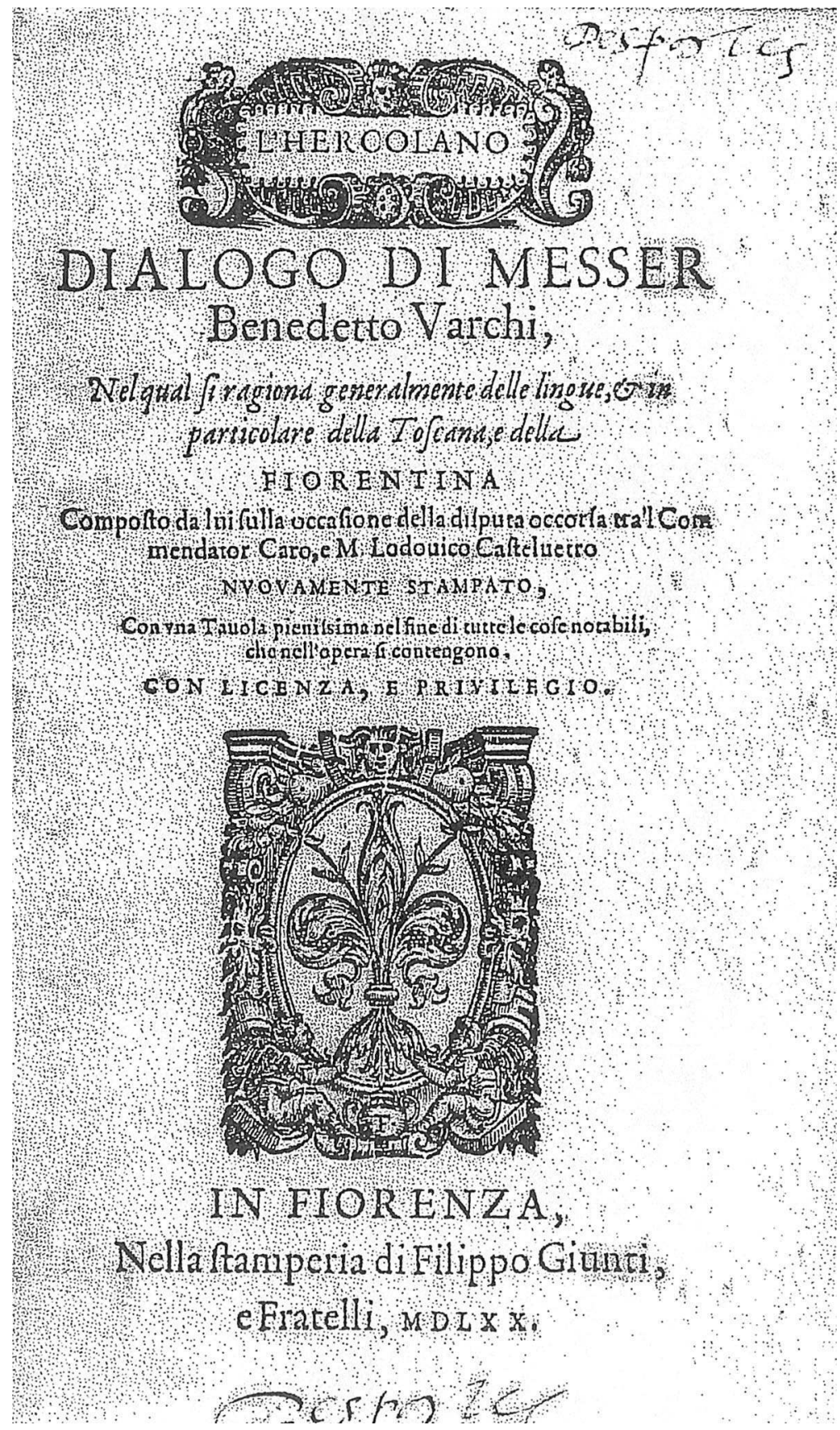

FIG. 1. 


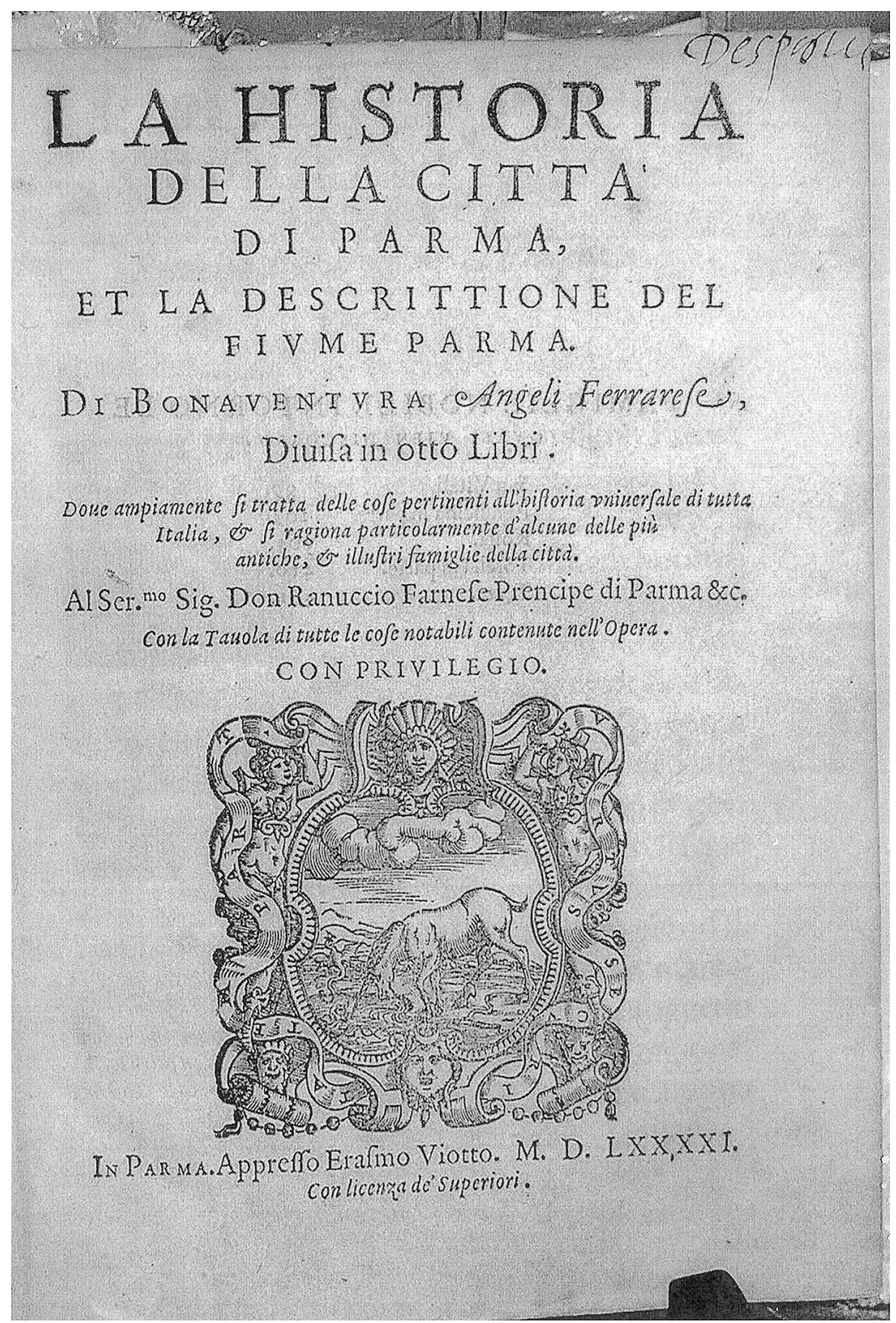

FIG. 2. 
François Rouget

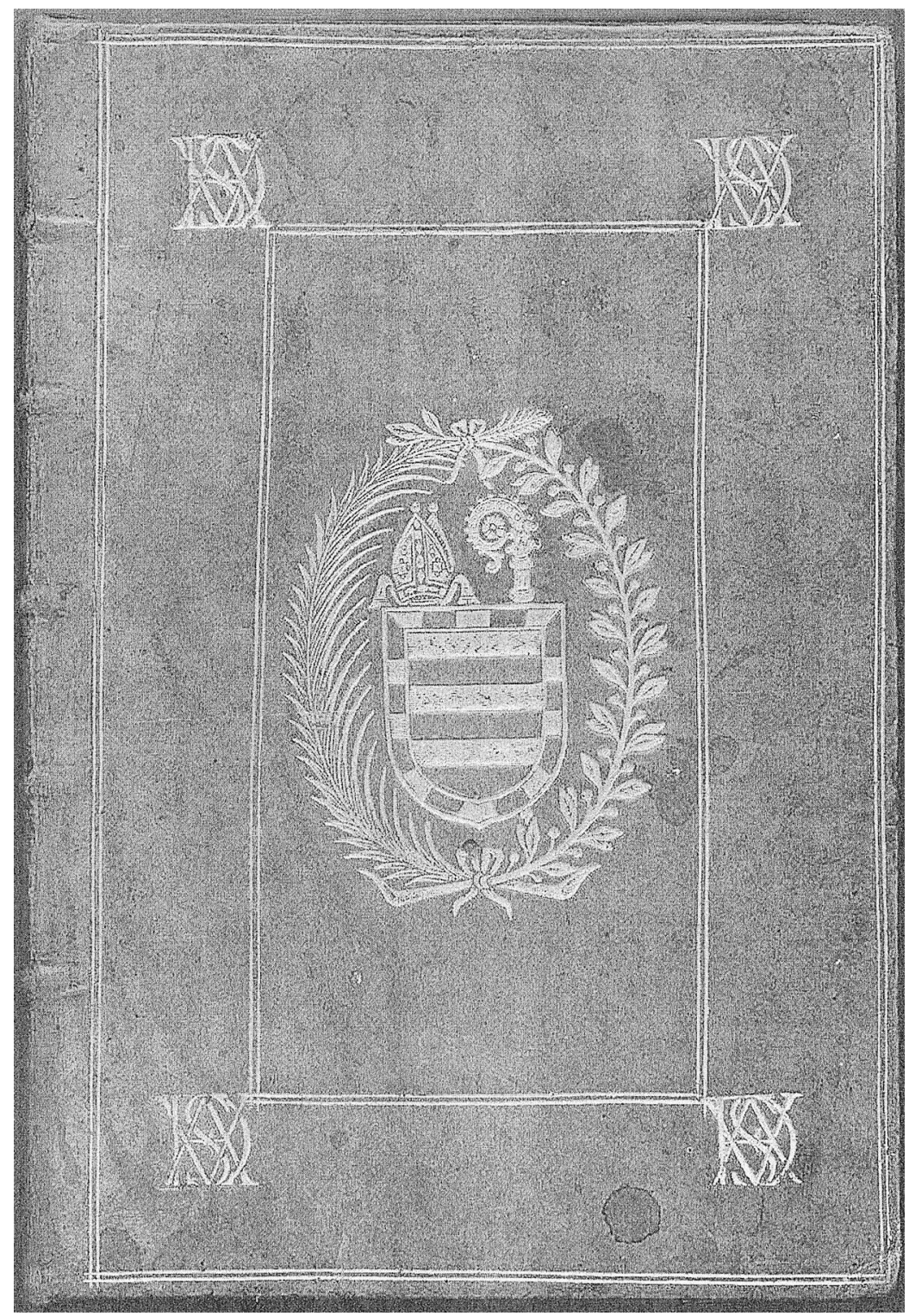

FIG. 3. 


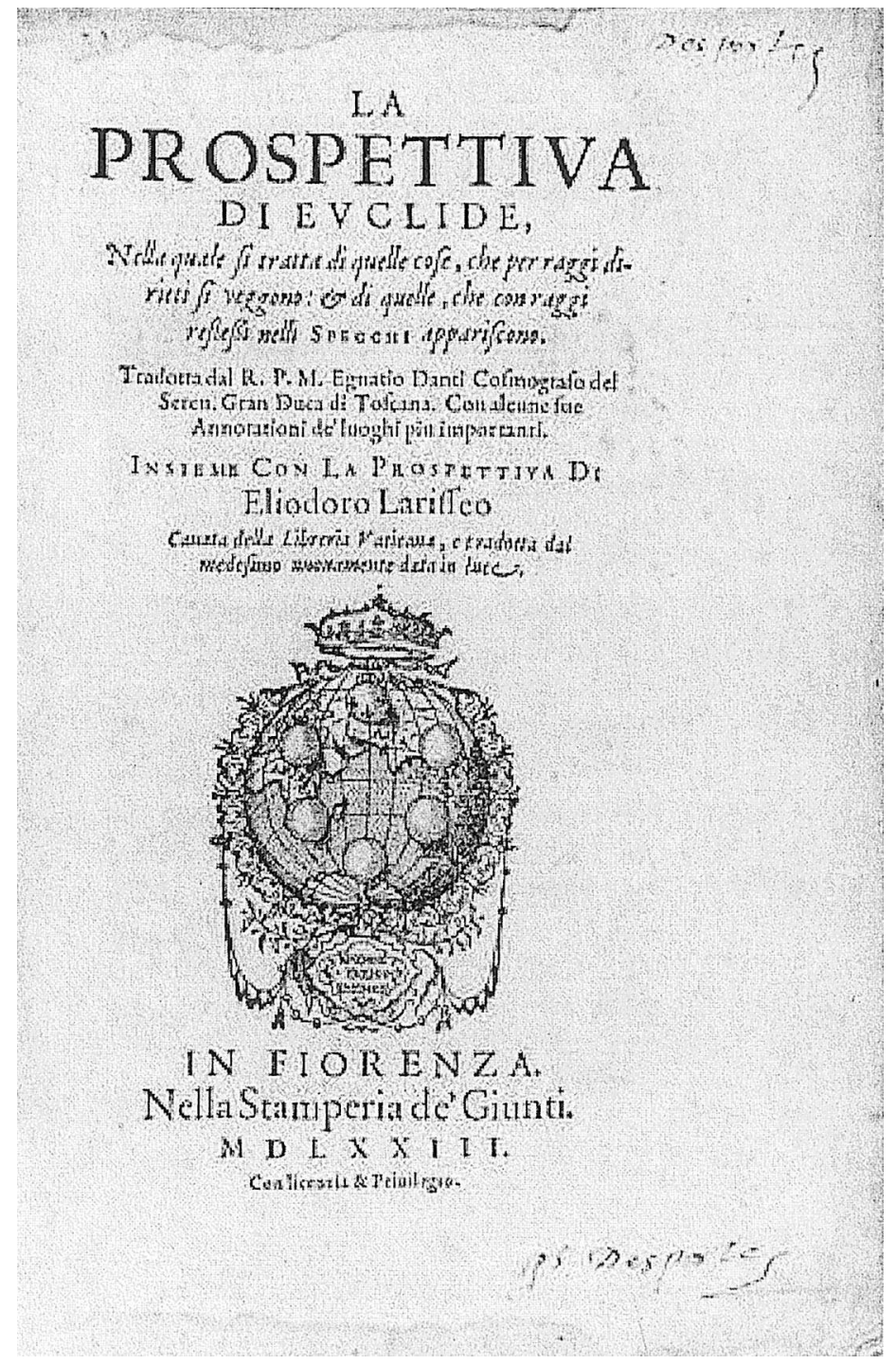

FIG. 4. 


\section{BARTHOLOMAEI} BOTTA PRASBYTERI

ETCANONICI PAPI-
SNIS DAVIDIAS. Goign pary. Soriot doju

(5) 1010

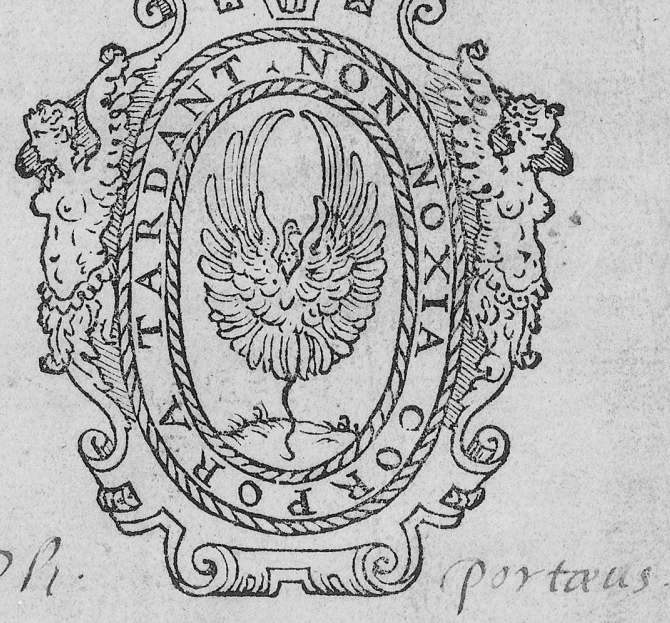

$$
P A P I E
$$

ApudHieronymum Bartholum . 1573.

FIG. 5. 


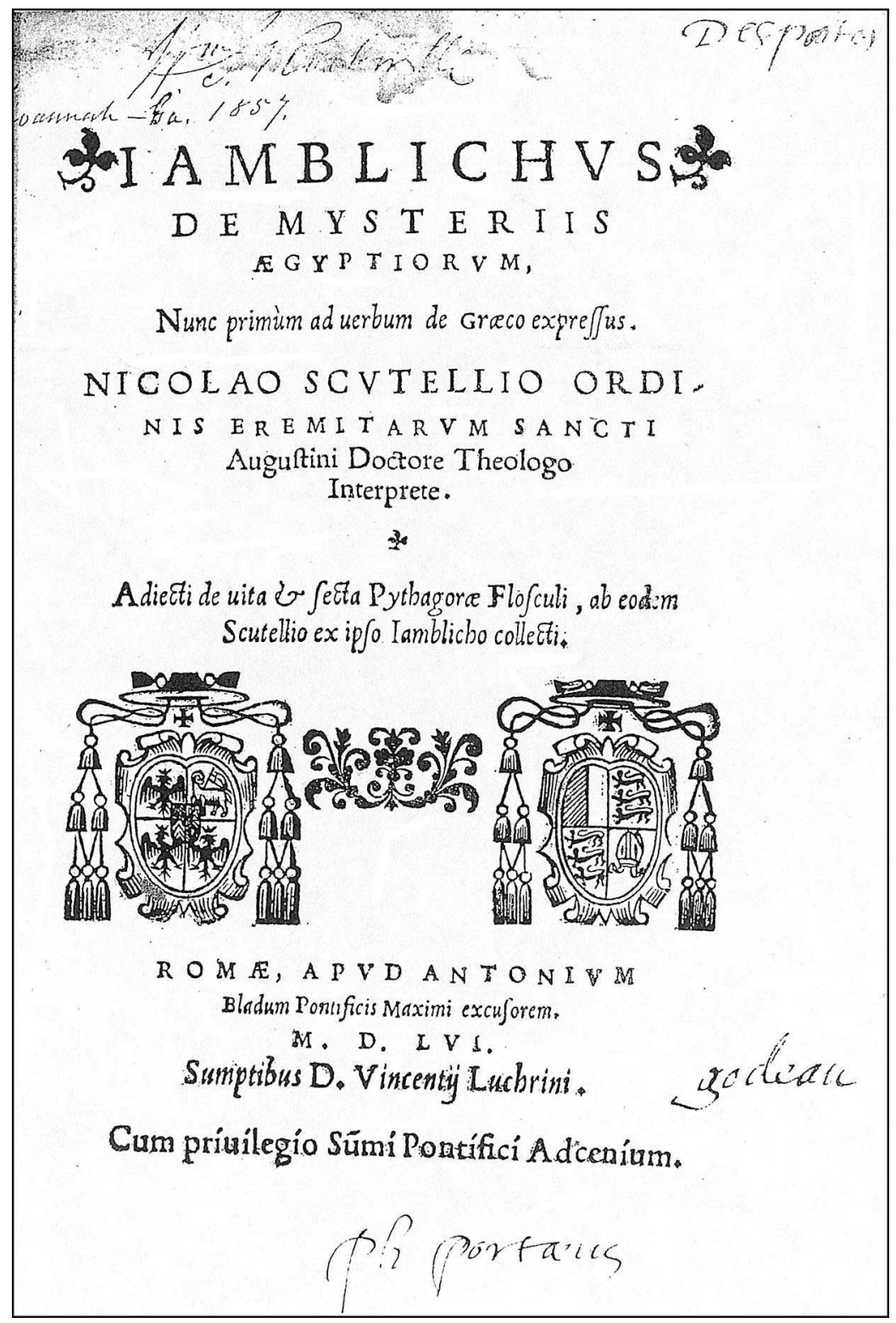

FIG. 6. 

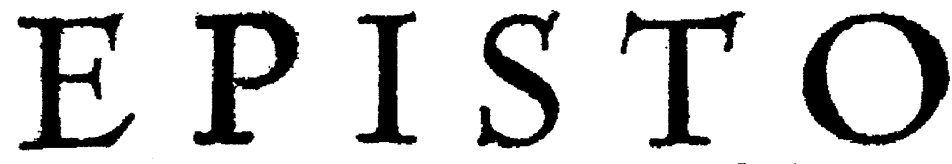

I A R N L E O NARII Aretini Libri octo, quarum fingulis ita fua funt argumenta, ut nulla ferè nō iuftus liber uideri, nom inarióp pof fit. Multum $\not \dot{z}$ eius temporis habent $\&$ factorum $\&$ confiliorum, qux notatu imprimis digna, alias memoria mandaca non reper tiuntur.

Despoztes

- SILEAE EXCVDEBAT HENRICVS PETRVS. 